\title{
Transpulmonary Closure of Giant Persistent Ductus Arteriosus under Cardiopulmonary Bypass and Normothermic Cardioplegia
}

\author{
Ujjwal K. Chowdhury ${ }^{1}$ Sukhjeet Singh ${ }^{1}$ Niwin George ${ }^{1}$ \\ Poonam Malhotra Kapoor ${ }^{2}$
}

Address for correspondence Ujjwal K. Chowdhury, MCh, Department of Cardiothoracic and Vascular Surgery, All India Institute of Medical Sciences, New Delhi 110029, India (e-mail: ujjwalchow@rediffmail.com).

J Card Crit Care TSS 2020;3:36-38
Abstract
Keywords
- giant ductus
arteriosus
- adult ductus
- cardiopulmonary
bypass
- transpulmonary
closure
- calcified ductus

A 25-year-old female patient with a giant, short, calcified, hypertensive, window ductus arteriosus underwent successful closure via transpulmonary approach under normothermic cardiopulmonary bypass without circulatory arrest using a Foley catheter for temporary occlusion.

\section{Introduction}

Despite 80 years of experience, correction of persistent ductus arteriosus remains a surgical challenge in the subset of patients with persistent ductus arteriosus presenting in adolescence and adulthood because of diminished elasticity, friability and/or calcification of vessel wall, previous infection causing ductal endarteritis, unusual anatomic features like giant ductus, wide ductus, short ductus, window ductus, aneurysmal ductus, and recurrent ductus. ${ }^{1-7}$

It is associated with dreaded complications related to surgical dissection and aortic cross-clamping like injury to the ductal tissue, distended pulmonary arteries, recurrent laryngeal nerve and large lymphatics. ${ }^{1-7}$

We present here-in the case of a 25-year-old female patient with a giant, short, calcified, hypertensive, window ductus arteriosus undergoing closure under normothermic cardiopulmonary bypass without circulatory arrest using Foley catheter for temporary occlusion ( - Fig. 1, - Fig. 2A-D).

The patient survived the operation. There was no bleeding, phrenic or left recurrent laryngeal nerve damage, chylothorax, or recanalization. Postoperative computerized tomographic angiography revealed complete ductal interruption with no residual shunt or ductal aneurysm ( - Fig. 3).

1. Primary median sternotomy is performed.

2. The thymus is dissected and the pericardium is opened in the midline in between stay sutures.

3. Aortobicaval cannulation: Patient is put on cardiopulmonary bypass and the left atrium is vented through right superior pulmonary vein on a partially filled heart to prevent intracardiac air suction ( - Video 1 ).

4. The great vessels are not isolated.

5. The pericardial reflection between the aorta and the main pulmonary artery is dissected and the aorta is isolated for later selective aortic cross-clamping.

6. Four stay sutures are placed on the distal part of main pulmonary artery for the performance of pulmonary arteriotomy at a later stage.

7. The aorta is cross-clamped; left atrial vent is placed on suction.

8. Myocardial protection is achieved by a combination of root cardioplegia and topical cooling. During ISSN 2457-0206.

\section{License terms}

()ㅜ(1) $\Theta \circledast$ 


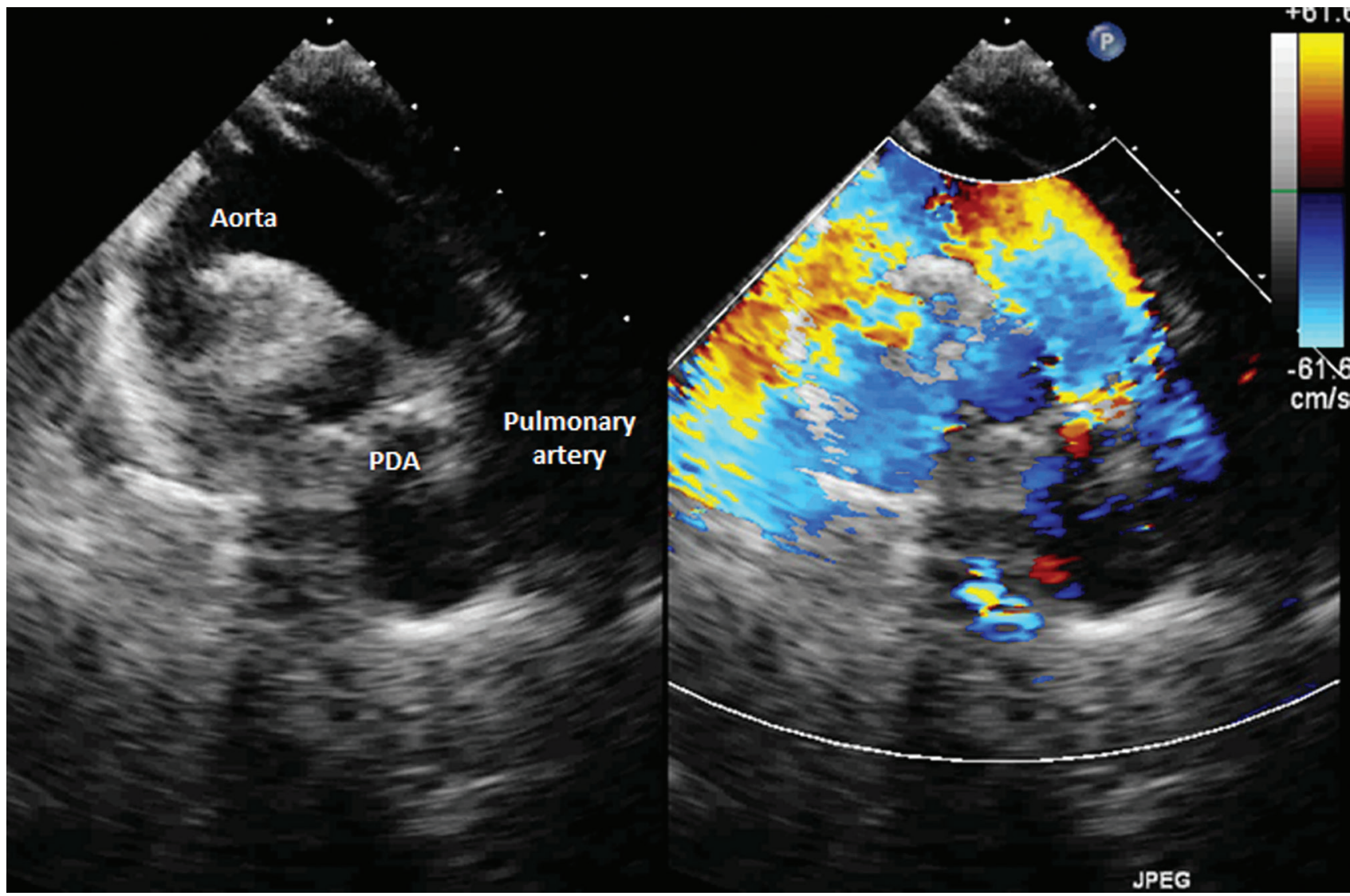

Fig. 1 Transthoracic two-dimensional color Doppler echocardiogram showing continuous wide flow from the aorta through the ductus to the pulmonary artery.
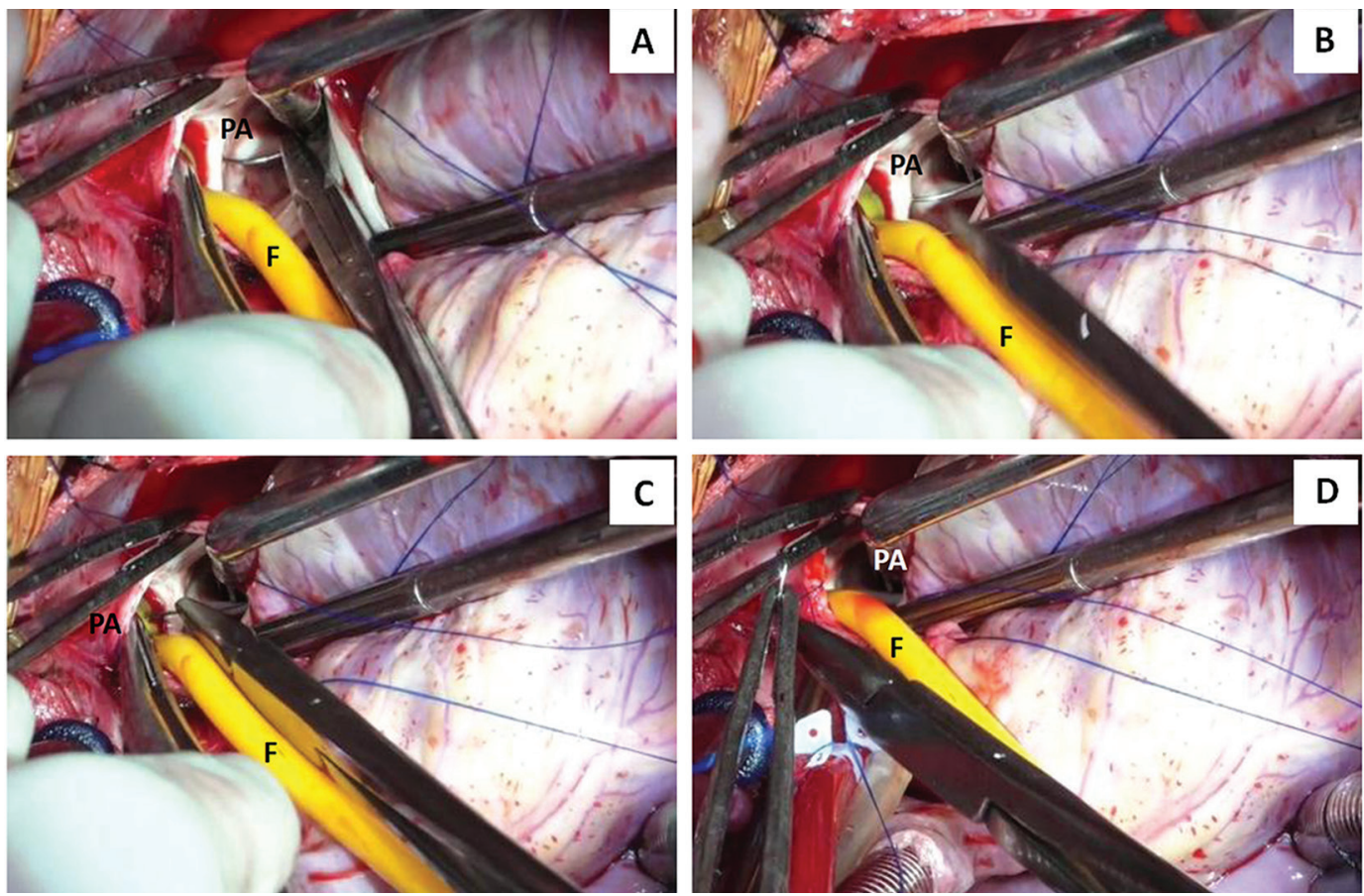

Fig. 2 (A-D) Surgical photographs showing step-by-step transpulmonary ductal closure under normothermic cardiopulmonary bypass and cardioplegic arrest. The aorta was individually cross-clamped. After cardioplegic arrest the pulmonary trunk was transversely opened in between stay sutures. The pump flows were transiently lowered to identify the pulmonary arterial end of the ductal orifice. A 20-Fr Foley catheter was inserted through the ductal orifice into the aorta. The ductal orifice was closed using multiple interrupted 4-0polypropylene suture buttressed with Teflon pledgets. The Foley catheter was advanced little deep within the aorta to avoid balloon rupture during suture placement. The balloon subsequently was deflated and withdrawn. 


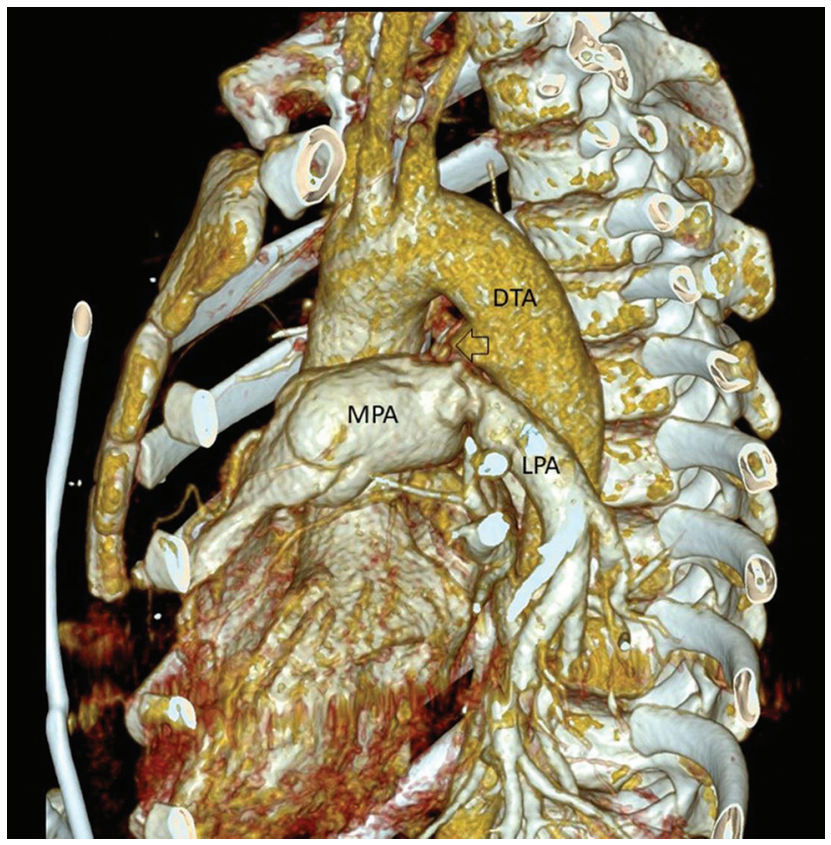

Fig. 3 Sagittal oblique computerized tomographic image showing the closed ductus arteriosus (arrow) with no flow between the aorta and pulmonary artery. DTA, descending thoracic aorta; LPA, left pulmonary artery; MPA, main pulmonary artery.

administration of cardioplegia digital compression was applied over the ductus area to prevent aortic run off, prevent cardiac distension, and to avoid myocardial rewarming (-Video 1).

9. The head end of the patient is lowered; the perfusionist is instructed to temporarily reduce the pump flow to 700 to $800 \mathrm{~mL}$ for proper visualization.

10. The ductus is occluded using a No.10 Foleys catheter with the balloon inflated and the pump flow was reverted back to normal.

11. The pulmonary arterial end of the ductus is occluded using three interrupted 5-0 pledgeted polypropylene sutures (Johnson and Johnson Ltd., Ethicon LLC, San Lorenzo, United States). Note the placement of three interrupted 5-0 polypropylene sutures over polytetrafluoroethylene pledgets (Bard Peripheral Vascular Inc., Tempe, Arizona, United States).

12. The pump flow is again transiently reduced to 700 to 800 $\mathrm{mL}$; the balloon of the Foleys catheter is deflated and the sutures are tied.

13. The pump flow is resumed back to full flow; the ductus is closed in two layers-an initial interrupted mattress and the second continuous running suture of 5-0 polypropylene (-Video 1 ).

14. The completeness of the ductus closure was assessed using table oxymetry and transesophageal echocardiography.

\section{Conclusion}

We conclude that appropriate surgical management of an adult patient with giant calcified and hypertensive window ductus should consist of occlusion with patch on cardiopulmonary bypass via transpulmonary approach using temporary balloon-occlusion. This method is safe, expedient, obviates the need for descending aortic cross-clamping, internal shunting, profound hypothermia, and circulatory arrest. It avoids dissection in the presence of ductal wall calcification, adhesions, thereby avoiding perioperative injury to the tense pulmonary artery and recurrent laryngeal nerve.

\section{Funding}

The authors received no financial support for the research, authorship and/or publication of this article.

\section{Conflict of Interest}

The author(s) declared no potential conflicts of interest with respect to the research, authorship and/or publication of the article.

\section{Video 1}

Transpulmonary closure of ductus arteriosus under cardiopulmonary bypass. Online content including video sequences viewable at: www.thieme-connect.com/ products/ejournals/html/10.1055/s-0039-1697547.

\section{References}

1 Arbatli H, Ozbek U, Demirsoy E, et al. Repair of recurrent patent ductus arteriosus in an adult with cardiopulmonary bypass. J Card Surg. 2003;18:17-9

2 Celermajer DS, Sholler GF, Hughes CF, Baird DK. Persistent ductus arteriosus in adults. A review of surgical experience with 25 patients. Med J Aust. 1991;155(4):233-236

3 Grunenfelder J, Bartram U, Van Praagh R, et al. The large window ductus: A surgical trap. Ann Thorac Surg. 1998;65:1790-1791

4 John S, Muralidharan S, Jairaj PS, Mani GK, et al. The adult ductus: review of surgical experience with 131 patients. J Thorac Cardiovasc Surg 1981;82(2):314-319

5 Oldham HN, Collins NP, Pierce GE, Sabiston DC, Blalock A. Giant patent ductus arteriosus. J Thorac Cardiovasc Surg 1964;47(3):331-336

6 Jatene MB, Abuchaim DCS, Tiveron MG, Tanamati C, Miura N, Riso A, et al. Surgical treatment of patent ductus arteriosus in adults. Braz J Cardiovasc Surg 2011;26(1):93-97

7 Sankhyan LK, Chowdhury UK, Gudala V, et al. Surgical management of the adult ductus, the giant ductus, the recanalized and calcified ductus using normothermic cardiopulmonary bypass without circulatory arrest: Review of 20 years' experience. Ann Short Reports 2018;1:1028 